\title{
Elevated carbohydrate antigen 125 post-operation as a prognostic marker in gastric cancer patients with stage II-III
}

\author{
Jingjing Li ${ }^{1,2,3}$, Qi Xu ${ }^{1,2,3}$, Cong Luo ${ }^{1,2,3}$, Lei Chen ${ }^{1,2,3}$, Jieer Ying ${ }^{1,2,3}$ \\ ${ }^{1}$ Institute of Cancer and Basic Medicine (ICBM), Chinese Academy of Sciences, Hangzhou, China; ${ }^{2}$ Department of Medical Oncology, Cancer \\ Hospital of the University of Chinese Academy of Sciences, Hangzhou, China; ${ }^{3}$ Department of Medical Oncology, Zhejiang Cancer Hospital, \\ Hangzhou, China \\ Contributions: (I) Conception and design: J Ying; (II) Administrative support: J Li; (III) Provision of study materials or patients: Q Xu; (IV) Collection \\ and assembly of data: C Luo; (V) Data analysis and interpretation: L Chen; (VI) Manuscript writing: All authors; (VII) Final approval of manuscript: \\ All authors. \\ Correspondence to: Jieer Ying. Department of Abdominal Medical Oncology, Zhejiang Cancer Hospital, Hangzhou 310022 , China. \\ Email: jieerying@aliyun.com.
}

\begin{abstract}
Background: Gastric cancer is one of the high occurrence cancers among gastrointestinal tumor. Further research should be carried out to find more prognostic factors, screening patients with poor prognosis, who need stronger treatment.

Methods: The clinicopathologic characteristic, the levels of tumor markers and survival of 105 patients who underwent gastrectomy with a D2 lymph node dissection in Zhejiang Cancer Hospital were collected and analyzed.

Results: In the 105 patients, there were 71 males, and the median age was 56 years (range, $21-83$ years). In 105 patients, 77 patients were diagnosed with non-cardia cancer, $84.8 \%$ patients were diagnosed of poorly differentiated adenocarcinoma, more than half of the patients were diagnosed of T3-4, and 92 patients had lymph node metastasis, while the clinical stage III patients were accounted for $85 \%$. Seventy point five percent patients were Borrmann III type. The median OS of the patients with increased preoperative serum CEA, CA125, CA19-9, AFP and combined were shorter than those patients with normal serum tumor markers, but without statistical significance $(\mathrm{P}=0.350, \mathrm{P}=0.498, \mathrm{P}=0.240, \mathrm{P}=0.578$ and $\mathrm{P}=0.219)$. The OS of patients with elevated levels of CEA, CA19-9, and AFP after operation were shorter than patients with decreased ones without statistical significance $(\mathrm{P}=0.321, \mathrm{P}=0.118$ and $\mathrm{P}=0.424)$. Elevated CA125 after operation was shorter than patients with decreased ones significantly $(\mathrm{P}<0.005)$.
\end{abstract}

Conclusions: Elevated of CA125 after operation was associated with poor OS in stage II-III GC patients.

Keywords: Gastric cancer; CEA; CA125; CA19-9; AFP

Submitted Nov 08, 2019. Accepted for publication Jul 27, 2020.

doi: $10.21037 /$ tcr-19-2427

View this article at: http://dx.doi.org/10.21037/tcr-19-2427

\section{Introduction}

Gastric cancer is one of the higher incidence cancers in gastrointestinal tumors, with obvious ethnic and geographical distributions (1). A total of 1,033,701 new cases were diagnosed in 2018, with an estimated 782,685 deaths, or $5.7 \%$ of the total number of cancer cases, accounting for $8.2 \%$ of total cancer deaths (1). More than
$70 \%$ of new cases and deaths occur in developing countries, particularly in East Asia, Eastern Europe and South America (1). In China, a total of 405,000 new cases were diagnosed in 2012, with an estimated death toll of 325,000 cases (2). For patients with removable gastric cancer, the prognosis is poor, the overall survival rate is 5 years, about $20-30 \%$ (3). Previous studies have shown that the prognostic factors identified were infiltration depth, lymph 
node metastasis, pathological types during tumor nodule metastasis (TNM), and whether to undergo postoperative treatment or not (4). However, some studies have shown that patients have heterogeneous clinical outcomes even at the same stage of the tumour.

This means that further research should be carried out to find more prognostic factors, screening patients with poor prognosis, who need stronger treatment. Tumor markers are defined as compounds produced by tumors or hosts in response to malignant neoplasms. They play an important role in cancer diagnosis, prognosis, treatment, and monitoring and have been widely used because of their inviolability and convenience. In recent years, CEA, CA125, CA19-9 and AFP have been widely used in gastric cancer. Some studies have reported that serum tumor marker levels can predict early and late prognosis in patients with gastric cancer stage $(5,6)$. Some studies have shown that single tumor markers can evaluate the therapeutic effect (7), and other studies have shown that comprehensive analysis of a variety of tumor markers for the diagnosis, prognosis and treatment of gastric cancer has a more important significance for patients (8). However, most studies focus only on the value of tumor markers at a given time and do not care about the effect of changes in tumor markers on the prognosis of patients with gastric cancer.

The purpose of this study was to evaluate the changes of CEA, CA125, CA19-9 and AFP before and after Phase III operation in gastric cancer phase II-III. We present the following article in accordance with the REMARK reporting checklist (available at http://dx.doi.org/10.21037/ tcr-19-2427).

\section{Methods}

The study was conducted in accordance with the Declaration of Helsinki (as revised in 2013). The study was approved by medical ethics committee of Zhejiang Cancer Hospital [No.: IRB-2020-152 (Ke)] and informed consent was taken from all the patients. This study is a retrospective review. Between January 2012 and December 2012, 105 patients at Zhejiang Cancer Hospital underwent D2 lymph node dissection, with a deadline of December 31, 2017 for data collection. Each patient has a histological diagnosis of adenocarcinoma. According to the 7th edition of AJCC staging, the postoperative pathological staging of all patients was II-III period. Each patient received more than 4 cycles of adjuvant chemotherapy, including S-1 single drug or XELOX, SOX combined drugs. Serum samples of tumor markers, including CEA, CA125, CA19-9 and AFP, were performed in each patient within 3-4 weeks before and after surgery. At the time of recruitment, personal data on the clinical characteristics of each patient, including age, gender, differentiation, tumor location, wall infiltration depth, lymph node metastasis, vascular infiltration and pathological tumor-node metastasis (pTNM) stage and collection of survival information from clinical records or family contact. The overall survival rate (OS) is defined as the time from diagnostic data to death or last access. Serum CEA, Ca19-9, CA125 and AFP levels were detected by electrochemical luminescence (ECL) (Abbott Laboratory, USA). As recommended by the manufacturer, the cutoff levels for CEA, CA125, CA19-9 and AFP are $5.0 \mathrm{ng} \cdot \mathrm{mL}^{-1}$, $35.0 \mathrm{U} \cdot \mathrm{mL}^{-1}, 37 \mathrm{U} \cdot \mathrm{mL}^{-1}$, and $8.1 \mathrm{ng} \cdot \mathrm{mL}^{-1}$. When the labeled serum level is higher than the critical value, the results are considered positive. Positive combined detection of four tumor markers is defined as one or more tumor markers that exceed the cutoff level. The data were analyzed using SPSS version 13.0. The continuous variables are represented by mean $\pm \mathrm{SD}$, and $t$-test and chi-squared Square test are used for comparison between the two groups. The predictive effect of each variable on survival was calculated by using Kaplan-Mayer method and logarithmic grade test. A P value of less than 0.05 is considered statistically significant.

\section{Results}

\section{Clinicopathologic characteristic}

Table 1 summarizes the clinicopathologic features of 105 patients. Of the 105 patients, $71(66.6 \%)$ were male, the median age was 55.2 (years at 21-83 years) and $58(55.2 \%)$ were under 60 years of age. In all cases, 28 (26.7\%) patients were treated with tachycardia adenocarcinoma and 77 $(73.3 \%)$ were diagnosed as non-myocardial cancer. Overall, $89(84.8 \%)$ of patients diagnosed with differentiated adenocarcinoma were moderately differentiated and well differentiated in 13 cases (12.4\%) and 3 (2.9\%). More than half (84.8) of the patients were diagnosed with T3-4 and 92 (88\%) lymph node metastasis, and clinical phase III patients accounted for $85 \%$. The 74 (70.5\%) and 25 (23.8\%) patients were Borman III and IV, respectively.

The relationship of preoperative serum TM status with Clinicopathologic characteristic CEA, CA125, CA199 and AFP before surgery were above the cutoff levels in $16.2 \%, 21 \%, 22.9 \%$ and $8.6 \%$ cases, respectively. A total of 56 people $(53.3 \%)$ showed positive for one or more 
Table 1 Clinical and pathological characteristics of the 105 patients

\begin{tabular}{|c|c|c|}
\hline Characteristic & $\mathrm{N}$ & $\%$ \\
\hline \multicolumn{3}{|l|}{ Age } \\
\hline$\leq 60$ & 58 & 55.2 \\
\hline$>60$ & 47 & 44.8 \\
\hline \multicolumn{3}{|l|}{ Sex } \\
\hline Male & 71 & 67.6 \\
\hline Female & 34 & 32.4 \\
\hline \multicolumn{3}{|l|}{ Tumor location } \\
\hline Cardia & 28 & 26.7 \\
\hline Non-cardia & 77 & 73.3 \\
\hline \multicolumn{3}{|l|}{ Histologic type } \\
\hline Poorly differentiated & 89 & 84.8 \\
\hline Moderately differentiated & 13 & 12.4 \\
\hline Well differentiated & 3 & 2.9 \\
\hline \multicolumn{3}{|l|}{ Depth of invasion } \\
\hline $\mathrm{T} 1-2$ & 16 & 15.2 \\
\hline T3-4 & 89 & 84.8 \\
\hline \multicolumn{3}{|l|}{ Nodes status } \\
\hline NO & 13 & 12.4 \\
\hline $\mathrm{N}+$ & 92 & 87.6 \\
\hline \multicolumn{3}{|l|}{ Stage } \\
\hline II & 20 & 19 \\
\hline III & 85 & 81 \\
\hline \multicolumn{3}{|l|}{ Borrmann } \\
\hline 1 & 5 & 4.8 \\
\hline 2 & 1 & 1 \\
\hline 3 & 74 & 70.5 \\
\hline 4 & 25 & 23.8 \\
\hline
\end{tabular}

tumor markers. Table 2 shows the relationship between preoperative levels and different clinicopathological parameters in each or combination of tumor manufacturers. Preoperative serum positive levels were 16.2\%, 21\%, 22.9\% and $8.6 \%$, respectively. There were $56(53.3 \%)$ patients who sowed positive for one or more tumor makers. CEA is more frequent in older patients $(\mathrm{P}=0.019)$, differentiation $(\mathrm{P}=0.012)$ patients and patients without lymph node involvement $(\mathrm{P}=0.002)$. Ca19-9 had a positive frequency in patients with advanced tumor stage $(\mathrm{P}=0.035)$. The positive levels of combined detection were related to the higher degree of tumor infiltration and advanced tumors $(\mathrm{P}=0.014$ and $\mathrm{P}=0.048)$.

\section{The relationship of preoperative serum TM status with OS}

The relationship between the level of a single tumor marker and the OS before surgery is shown in Figure 1. In the last follow-up, about half of the cases were still alive, with a 5 -year survival rate of $49.5 \%$. The mortality rates for normal levels of pre-operative CEA, CA125, CA19-9 and AFP were 50\% (444/88), 50.6 (422/83), 48.1\% (39/81) and $50 \%$ (48/96), respectively. The mortality rates of preoperative CEA, CA125, CA19-9 and AFP were $52.9 \%$ (9/17), 50\% (11/22), 52.9\% (14/24) and 52.9\% (5/9), respectively. In some studies, tumor markers include CEA, CA125, CA19-9 and AFP as prognostic factors for gastric cancer OS. In this study, the median OS was 36, 28, 30 and 36 months in patients with elevated preoperative serum CEA, CA125, CA19-9 and AFP levels, which was shorter than that of patients with normal serum tumor markers, but had no statistical importance $(\mathrm{P}=0.350, \mathrm{P}=0.498, \mathrm{P}=0.240$ and $\mathrm{P}=0.578)$. Figure 2 shows the relationship between CEA, CA125, CA19-9, and AFP combined with tumor markers and OS. The OS time in patients with negative tumor markers was longer than that of any positive tumor marker, but not statistically significant $(\mathrm{P}=0.219)$.

\section{The relationship of the changes of serum TM status between pre and post operation with OS}

Figure 3 shows the relationship between preoperative and postoperative changes in tumor marker levels and OS. The proportion of patients with elevated CEA, CA125, CA199 and AFP after operation was 30.5\%, 31.4\%, 40\% and $50.5 \%$, respectively. Patients with elevated CEA levels, CA125, CA19-9, AFP had a mortality rate of 53.1\% (17/32), $72.7 \%(24 / 33), 59.5 \%(25 / 42)$ and $54.7 \%$ (29/53), higher than cases of decreased tumor marker levels (49.3\%, 40.3\%, $44,4 \%$ and $46.1 \%$ ). The OS for patients with elevated CEA is $3-39$ months (median 34.3 months). In patients with a decline in CEA levels, the OS time was longer, but not statistically significant $(\mathrm{P}=0.321)$. $\mathrm{Ca} 19-9(\mathrm{P}=0.118)$ and AFP ( $\mathrm{P}=0.424)$ also found the situation. The OS of CA125 elevated patients was 3-36 (median 26.6 months), which was shorter than that of patients with decreased CA125 level after operation (median 39.8 months, $\mathrm{P}<0.005$ ). 
Table 2 Association of preoperative status of tumor markers with clinicopathological parameters

\begin{tabular}{|c|c|c|c|c|c|c|c|c|c|c|c|}
\hline Characteristic & Cases (N) & $\begin{array}{l}\text { CEA(+), } \\
N(\%)\end{array}$ & $P$ value & $\begin{array}{l}\text { CA125(+), } \\
\text { N (\%) }\end{array}$ & $P$ value & $\begin{array}{l}\text { CA19-9(+), } \\
\quad \mathrm{N}(\%)\end{array}$ & $P$ value & $\begin{array}{l}\text { AFP(+), } \\
N(\%)\end{array}$ & $P$ value & $\begin{array}{l}\text { Combined } \\
\text { MT, N (\%) }\end{array}$ & $P$ value \\
\hline \multicolumn{12}{|l|}{ Age } \\
\hline$\leq 60$ & 58 & 9 & 0.593 & 13 & 0.683 & 11 & 0.291 & 4 & 0.496 & 27 & 0.122 \\
\hline$>60$ & 47 & 8 & & 9 & & 13 & & 5 & & 29 & \\
\hline \multicolumn{12}{|l|}{ Sex } \\
\hline Female & 34 & 4 & & 6 & & 9 & & 5 & & 19 & \\
\hline \multicolumn{12}{|l|}{ Tumor location } \\
\hline Cardia & 28 & 6 & 0.38 & 4 & 0.311 & 7 & 0.753 & 3 & 0.636 & 17 & 0.361 \\
\hline \multicolumn{12}{|l|}{ Histologic type } \\
\hline $\begin{array}{l}\text { Moderately/well } \\
\text { differentiated }\end{array}$ & 16 & 1 & & 2 & & 1 & & 1 & & 9 & \\
\hline \multicolumn{12}{|l|}{ Depth of invasion } \\
\hline T1-2 & 16 & 2 & 0.663 & 2 & 0.367 & 1 & 0.086 & 0 & 0.183 & 4 & 0.014 \\
\hline T3-4 & 89 & 15 & & 20 & & 23 & & 9 & & 52 & \\
\hline \multicolumn{12}{|l|}{ Nodes status } \\
\hline No & 13 & 6 & 0.002 & 4 & 0.353 & 1 & 0.164 & 0 & 0.238 & 8 & 0.526 \\
\hline $1+2+3$ & 80 & 16 & 0.058 & 17 & 0.893 & 18 & 0.876 & 7 & 0.907 & 43 & 0.878 \\
\hline 4 & 25 & 1 & & 5 & & 6 & & 2 & & 13 & \\
\hline
\end{tabular}

\section{Discussion}

High incidence of gastric cancer, high mortality rates and limited treatment options remain a major health problem worldwide (1). The relative survival rate of five years of age standardization was reported to be $27.4 \%$ (95\% CI: $26.7-18.1 \%$ ) in 2003-2005 based on population-based cancer registration data in China (9). In this study, the 5 -year survival rate of two stage III gastric cancer patients was $49.5 \%$, which was better than the national data. Most of the patients in this study came from developed regions and received D2 lymph node dissection, followed by more than
4 cycles of adjuvant chemotherapy, including S-1 singledrug or capecitabine and oxaliplatin or S-1 and oxaliplatin combined in our hospital.

These patients have good compliance, so they have a better prognosis. Surgery is still the main treatment for gastric cancer, especially in early gastric cancer patients. Long-term follow-up data from the Dutch gastric cancer group test confirmed the survival benefits of D2 lymph node dissection (10). The 15 -year operating system rates for the D1 and D2 groups were $21 \%$ and $29 \%(P=0.34)(11)$, respectively. D2 lymph node dissection was also associated 
A

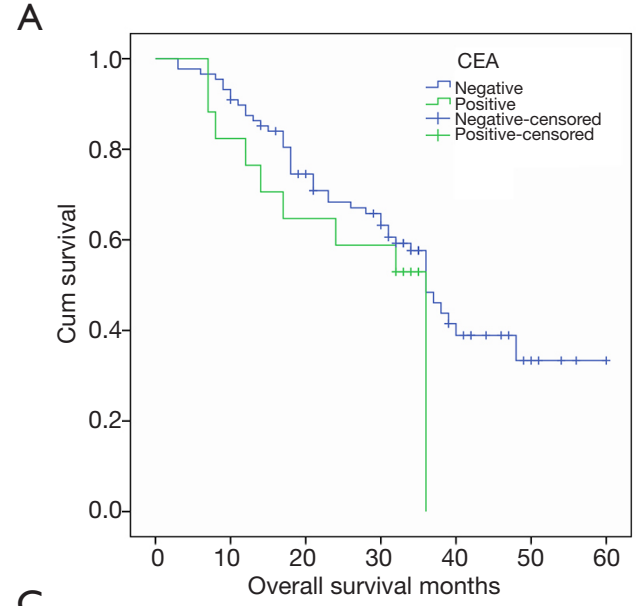

$\mathrm{C}$

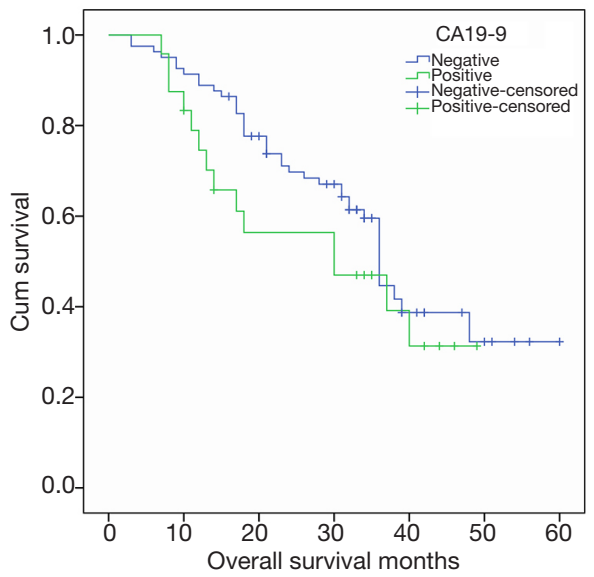

B
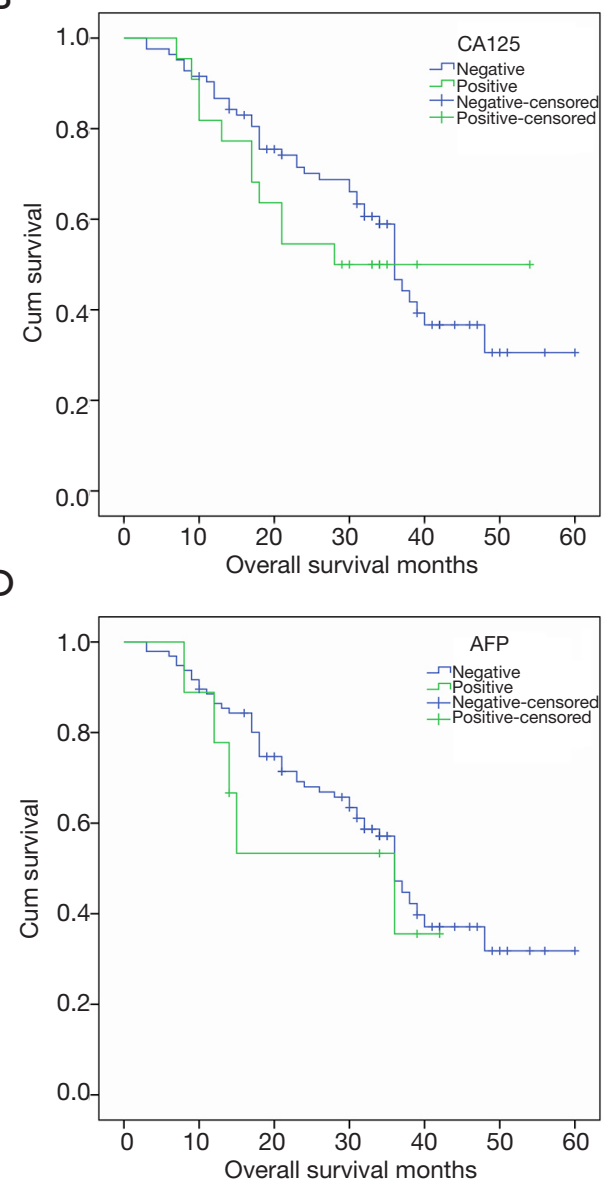

Figure 1 Survival curves of patients according to CEA, CA125, CA19-9, and AFP preoperation serum positivity. The difference between CEA-negative patients and CEA-positive patients (A), CA125-negative patients and CA125-positive patients (B), CA19-9-negative patients and CA19-9-positive patients (C), AFP-negative patients and AFP-positive patients (D) were not statistically significant $\mathrm{P}=0.350, \mathrm{P}=0.498$, $\mathrm{P}=0.240$ and $\mathrm{P}=0.578$ ).

with lower local rates (12\% to $22 \%$ ) and regional recurrence (13\% to $19 \%)$. More importantly, the mortality associated with gastric cancer in the D2 group was significantly lower than in the D1 group (37\% and 48\%, respectively). Two other studies from Western countries also reported better results for D2 lymph node dissection based on the recommendations of the Japanese Research Society for Gastric Cancer $(12,13)$. In East Asia, D2 lymph node dissection is a commonly recommended surgical method. In this study, all patients underwent D2 lymph node dissection. NCCN Clinical Practice Guidelines in Oncology Version 1. 2018 suggested that patients who have undergone primary D2 lymph node dissection could receive capecitabine and oxaliplatin as postoperative chemotherapy according to the result of study CLASSIC (14). Another study showed that S-1 plus docetaxel postoperative adjuvant therapy had been shown to improve the overall survival rate and nonrecurrence survival of patients with stage III gastric cancer receiving D2 gastrectomy (15). In this study, all patients underwent D2 lymph node dissection and were then treated with more than 4 cycles of adjuvant chemotherapy, including S-1 single drug or capecitabine and oxaliplatin or S-1 and oxaliplatin combined drugs. However, some patients will relapse in the following years. Finding patients at high risk of recurrence, followed by vigorous treatment, can improve the prognosis of patients with gastric cancer.

Serum tumor markers were simple to detect, noninvasive, the measurement process was mature and 


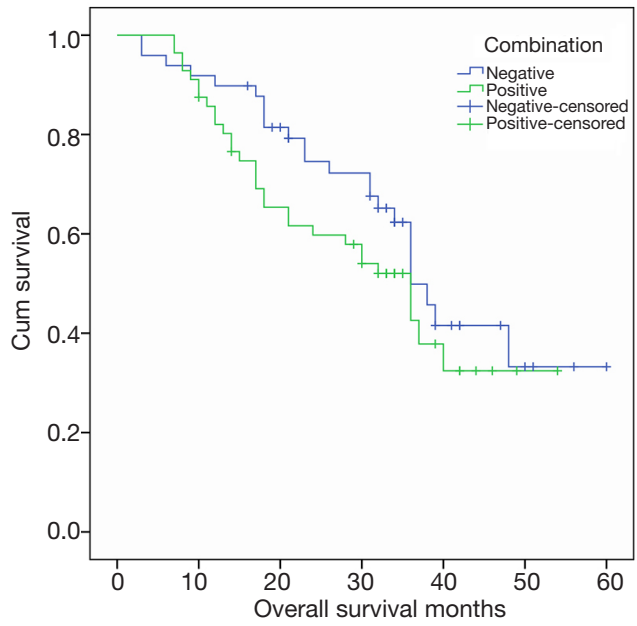

Figure 2 Survival curves of patients according to combination of CEA, CA125, CA19-9, and AFP preoperation serum positivity. The difference between all-negative patients and more one of tumor makers positive patients was not statistically significant $(\mathrm{P}=0.219)$.

the repeatability was good. They are considered to be monitoring markers for tumor prognosis and treatment evaluation. In this study, the prognostic value of CEA, CA125, CA19-9 and AFP changes before and after operation in phase two Stage III gastric cancer patients was discussed. Previous studies have reported that serum levels in the serum of patients with gastric cancer, such as CEA, CA125, CA19-9 and AFP, were elevated not only at an advanced stage, but also in the early stages. The positive rate of pretreatment of tumor markers is different in previous studies. CEA positive rates are usually between $15.8 \%$ and $57.6 \%$, CA125 between $18.2 \%$ and $53.9 \%$, CA19-9 between $19.1 \%$ and $50 \%$, AFP between $5.4 \%$ and $11.8 \%(16-18)$, and our findings on the percentage of positive markers are in these Within the range (CEA: 16.2\%; CA125: 21.0\%; CA19-9: 22.9\%; and AFP: 8.6\%), our research positive rate is at a lower level because there is no transfer of patients in our research. As mentioned earlier, serum CEA levels are associated with tumor depth, lymph node involvement (19). Feng's research shows that the increase in CEA levels is an independent risk factor for the poor prognosis of early gastric cancer (6). A metaanalysis showed that CEA protein and mRNA levels in peritoneal lavage were associated with peritoneal recurrence after radical gastrectomy (20). In order to understand the correlation between the pretreatment status of tumor markers and different clinicopathological parameters, we conducted a chi-square test and found that CEA positive was related to differentiation level and lymph node involvement. Previous studies have confirmed that the preoperative positive serum of CA125 is associated with poor prognosis in patients with gastric cancer (21). Kim and others proved that in 679 patients undergoing resection, the serum CA125 value was an independent prognostic risk factor, and the risk rate of recurrence of gastric cancer was $2.431(22)$.

However, no link was found between CA125 levels and clinicopathological features. Previous studies have reported that patients with high levels of serum CA19-9 before surgery are much more likely to exhibit lymphatic infiltration, perineal infiltration, advanced tumors and recurrence than patients with normal preoperative serum CA19-9 levels $(23,24)$. A meta-analysis of 11,408 patients with gastric cancer showed that elevated serum CA199 levels were associated with low levels of prognosis (25). Our findings confirm that it is associated with advanced tumor periods. Elevated AFP levels are more likely to show liver metastasis and poor gastric prognosis cancer $(26,27)$, according to reports. However, in our study, no link was found between AFP levels and Clinicopathological features. We also found that the positive detection of four tumor markers was related to the improvement of tumor infiltration degree and the advancement of tumor staging.

Contrary to these studies, in this outcome, we found a trend in preoperative CEA, CA125, CA19-9 and AFP, and even combined detection as a prognostic factor in the second to III phases of gastric cancer, but without statistical significance. The possible reason is that all patients in the study underwent D2 lymph node dissection and more than 4 cycles of adjuvant chemotherapy, including S-1 single drug or capecitabine and oxaliplatin or S-1 and oxaliplatin combined drugs, which can alter and improve the prognosis of these patients. The clinical evaluation of the changes of CEA, CA125, CA19-9 and AFP before and after operation in these patients was analyzed. We found that elevated postoperative CA125 was associated with poor OS in stage II-III GC patients. This is because the increase in CA125 is associated with peritoneal metastasis $(5,28)$, and the prognosis of patients with peritoneal metastasis is worse than that of liver, lung and other metastases (29). The peritoneum is the most common site for metastasis and recurrence in patients with gastric cancer (30).

In order to improve the OS of these patients, whether 

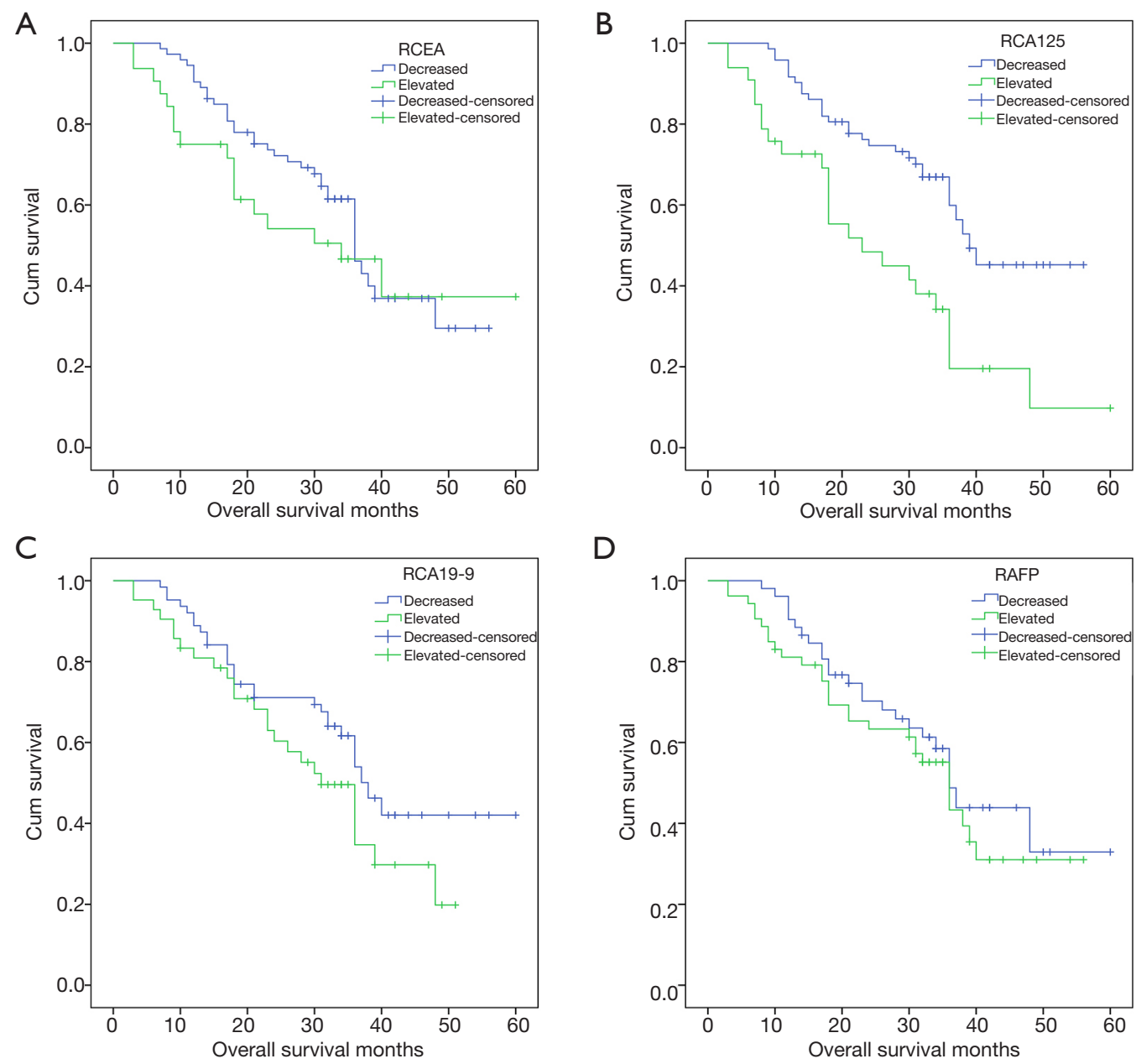

Figure 3 Survival curves of patients according to the changes of CEA, CA125, CA19-9, AFP serum status between pre and post operation. (A) The difference between CEA-decreased patients and CEA-elevated patients was not statistically significant $(\mathrm{P}=0.321)$. (B) The difference between CA125-decreased patients and CA125-elevated patients was statistically significant $(\mathrm{P}<0.005)$. (C) The difference between C199-decreased patients and CA19-9-elevated patients was not statistically significant $(\mathrm{P}=0.118)$. (D) The difference between AFP-decreased patients and AFP-elevated patients was not statistically significant $(\mathrm{P}=0.424)$.

or not patients with elevated postoperative CA125 need stronger adjuvant chemotherapy, or even intraperitoneal chemotherapy, this requires further study of more accurate research.

In this study, prejudice is inevitable due to some potential limitations. This retrospective study was conducted in a single institution and did not take into account a number of potential common factors related to tumor makers in all analyses. Our results need more evidence to support it. In summary, postoperative CA125 elevation was associated with poor OS in two-III GC patients. Larger, forward-looking and randomized studies are needed to confirm these findings.

\section{Acknowledgments}

We thank all the clinical investigators of the selected studies and patients involved in these studies.

Funding: This work was supported by the Zhejiang Science and Technology Office Project Foundation, no. 2016KYA050.

\section{Footnote}

Reporting Checklist: The authors have completed the REMARK reporting checklist. Available at http://dx.doi. org/10.21037/tcr-19-2427 
Data Sharing Statement: Available at http://dx.doi. org/10.21037/tcr-19-2427

Conflicts of Interest: All authors have completed the ICMJE uniform disclosure form (available at http://dx.doi. org/10.21037/tcr-19-2427). The authors have no conflicts of interest to declare.

Ethical Statement: The authors are accountable for all aspects of the work in ensuring that questions related to the accuracy or integrity of any part of the work are appropriately investigated and resolved. The study was conducted in accordance with the Declaration of Helsinki (as revised in 2013). The study was approved by medical ethics committee of Zhejiang Cancer Hospital [No.: IRB2020-152 (Ke)] and informed consent was taken from all the patients.

Open Access Statement: This is an Open Access article distributed in accordance with the Creative Commons Attribution-NonCommercial-NoDerivs 4.0 International License (CC BY-NC-ND 4.0), which permits the noncommercial replication and distribution of the article with the strict proviso that no changes or edits are made and the original work is properly cited (including links to both the formal publication through the relevant DOI and the license). See: https://creativecommons.org/licenses/by-nc-nd/4.0/.

\section{References}

1. Bray F, Ferlay J, Soerjomataram I, et al. Global cancer statistics 2018: GLOBOCAN estimates of incidence and mortality worldwide for 36 cancers in 185 countries. CA Cancer J Clin 2018;68:394-424.

2. Torre LA, Bray F, Siegel RL, et al. Global cancer statistics, 2012. CA Cancer J Clin 2015;65:87-108.

3. Reddavid R, Sofia S, Chiaro P, et al. Neoadjuvant chemotherapy for gastric cancer. Is it a must or a fake? World J Gastroenterol 2018;24:274-89.

4. Liu JY, Peng CW, Yang XJ, et al. The prognosis role of AJCC/UICC 8(th) edition staging system in gastric cancer, a retrospective analysis. Am J Transl Res 2018;10:292-303.

5. Huang C, Liu Z, Xiao L, et al. Clinical Significance of Serum CA125, CA19-9, CA72-4, and Fibrinogen-toLymphocyte Ratio in Gastric Cancer With Peritoneal Dissemination. Front Oncol 2019;9:1159.

6. Feng F, Tian Y, Xu G, et al. Diagnostic and prognostic value of CEA, CA19-9, AFP and CA125 for early gastric cancer. BMC Cancer 2017;17:737.

7. Acharya A, Markar SR, Matar M, et al. Use of Tumor Markers in Gastrointestinal Cancers: Surgeon Perceptions and Cost-Benefit Trade-Off Analysis. Ann Surg Oncol 2017;24:1165-73.

8. Lin JX, Wang W, Lin JP, et al. Preoperative Tumor Markers Independently Predict Survival in Stage III Gastric Cancer Patients: Should We Include Tumor Markers in AJCC Staging? Ann Surg Oncol 2018;25:2703-12.

9. Zhang SW, Yang ZX, Zheng RS, et al. Incidence and mortality of stomach cancer in China, 2013. Zhonghua Zhong Liu Za Zhi 2017;39:547-52.

10. Park SH, Son T, Seo WJ, et al. Prognostic Impact of Extended Lymph Node Dissection versus Limited Lymph Node Dissection on pN0 Proximal Advanced Gastric Cancer: a Propensity Score Matching Analysis. J Gastric Cancer 2019;19:212-24.

11. Songun I, Putter H, Kranenbarg EM, et al. Surgical treatment of gastric cancer: 15-year follow-up results of the randomised nationwide Dutch D1D2 trial. Lancet Oncol 2010;11:439-49.

12. Degiuli M, Sasako M, Ponti A, et al. Morbidity and mortality in the Italian Gastric Cancer Study Group randomized clinical trial of D1 versus D2 resection for gastric cancer. Br J Surg 2010;97:643-9.

13. van de Velde CJ. Randomized clinical trial comparing survival after D1 or D2 gastrectomy for gastric cancer. Br J Surg 2014;101:31-2.

14. Noh SH, Park SR, Yang HK, et al. Adjuvant capecitabine plus oxaliplatin for gastric cancer after D2 gastrectomy (CLASSIC): 5-year follow-up of an open-label, randomised phase 3 trial. Lancet Oncol 2014;15:1389-96.

15. Yoshida K, Kodera Y, Kochi M, et al. Addition of Docetaxel to Oral Fluoropyrimidine Improves Efficacy in Patients With Stage III Gastric Cancer: Interim Analysis of JACCRO GC-07, a Randomized Controlled Trial. J Clin Oncol 2019;37:1296-304.

16. Jing J, Ge M, Yang Z, et al. Spatial distribution characteristics of tumor marker CA724 reference values in China. Cancer Med 2019;8:4465-74.

17. Ghaderi B, Moghbel H, Daneshkhah N, et al. Clinical Evaluation of Serum Tumor Markers in the Diagnosis of Gastric Adenocarcinoma Staging and Grading. J Gastrointest Cancer 2019;50:525-9.

18. Kim JH, Jun KH, Jung H, et al. Prognostic Value of Preoperative Serum Levels of Five Tumor Markers 
(Carcinoembryonic Antigen, CA19-9, Alpha-

fetoprotein, CA72-4, and CA125) in Gastric Cancer.

Hepatogastroenterology 2014;61:863-9.

19. Wang W, Seeruttun SR, Fang C, et al. Prognostic Significance of Carcinoembryonic Antigen Staining in Cancer Tissues of Gastric Cancer Patients. Ann Surg Oncol 2016;23:1244-51.

20. Xiao Y, Zhang J, He X, et al. Diagnostic values of carcinoembryonic antigen in predicting peritoneal recurrence after curative resection of gastric cancer: a meta-analysis. Ir J Med Sci 2014;183:557-64.

21. Namikawa T, Kawanishi Y, Fujisawa K, et al. Serum carbohydrate antigen 125 is a significant prognostic marker in patients with unresectable advanced or recurrent gastric cancer. Surg Today 2018;48:388-94.

22. Kim DH, Yun HY, Ryu DH, et al. Preoperative CA 125 is significant indicator of curative resection in gastric cancer patients. World J Gastroenterol 2015;21:1216-21.

23. Kotzev AI, Draganov PV. Carbohydrate Antigen 19-9, Carcinoembryonic Antigen, and Carbohydrate Antigen 72-4 in Gastric Cancer: Is the Old Band Still Playing? Gastrointest Tumors 2018;5:1-13.

24. Sisik A, Kaya M, Bas G, et al. CEA and CA 19-9 are still valuable markers for the prognosis of colorectal and gastric cancer patients. Asian Pac J Cancer Prev 2013;14:4289-94.

25. Song YX, Huang XZ, Gao P, et al. Clinicopathologic and Prognostic Value of Serum Carbohydrate Antigen 19-9 in Gastric Cancer: A Meta-Analysis. Dis Markers 2015;2015:549843

26. Chen $Y, Q u$ H, Jian M, et al. High level of serum AFP is an independent negative prognostic factor in gastric cancer. Int J Biol Markers 2015;30:e387-93.

27. Zuo CT, Ju Q. An analysis of clinical characteristics and prognosis for patients with serum alpha-fetoproteinpositive gastric cancer. Minerva Med 2015;106:185-91.

28. Zhou P, Qu H, Shi H, et al. Predictive value of CA125 in peritoneal metastasis and prognosis of patients with gastric carcinoma. Zhonghua Wei Chang Wai Ke Za Zhi 2014;17:1027-30.

29. Chau I, Norman AR, Cunningham D, et al. Multivariate prognostic factor analysis in locally advanced and metastatic esophago-gastric cancer--pooled analysis from three multicenter, randomized, controlled trials using individual patient data. J Clin Oncol 2004;22:2395-403.

30. Nashimoto A, Akazawa K, Isobe $Y$, et al. Gastric cancer treated in 2002 in Japan: 2009 annual report of the JGCA nationwide registry. Gastric Cancer 2013;16:1-27.
Cite this article as: $\mathrm{Li} \mathrm{J,} \mathrm{Xu} \mathrm{Q}$, Luo C, Chen L, Ying J. Elevated carbohydrate antigen 125 post-operation as a prognostic marker in gastric cancer patients with stage II-III. Transl Cancer Res 2020;9(9):5200-5208. doi: 10.21037/tcr-192427 